\title{
Design optimization of shape memory alloy active structures using the R-phase transformation
}

\author{
Matthijs Langelaar* and Fred van Keulen \\ Department of Precision and Microsystems Engineering, \\ Delft University of Technology, Mekelweg 2, 2628 CD Delft, the Netherlands
}

\begin{abstract}
This article illustrates the opportunities that combining computational modeling and systematic design optimization techniques offer to facilitate the design process of shape memory alloy (SMA) structures. Focus is on shape memory behavior due to the R-phase transformation in $\mathrm{Ni}$ - $\mathrm{Ti}$, for which a dedicated constitutive model is formulated. In this paper, efficient topology and shape optimization procedures for the design of SMA devices are described. In order to achieve fast convergence to optimized designs, sensitivity information is computed to allow the use of gradient-based optimization algorithms. The effectiveness of the various optimization procedures is illustrated by numerical examples, including the design of a miniature SMA gripper and a steerable SMA active catheter. It is shown that design optimization enables designers of SMA structures to systematically enhance the performance of SMA devices for a variety of applications.
\end{abstract}

Keywords: Shape memory alloys, Ni-Ti, R-phase, topology optimization, shape optimization, sensitivity analysis, active catheter

\section{INTRODUCTION}

Shape memory alloys (SMAs) are attractive materials for active smart structures, because of the high work density these materials offer. Their actuation properties originate from a solid state phase transformation, which occurs under local temperature changes and/or stress state variations. This transformation is associated with a transformation strain that can be utilized for actuation. In many cases, an electric current is used to locally heat the material. SMA actuators are well suited for situations requiring relatively large deflections, combined with substantial mechanical loading. ${ }^{1,2}$ Depending on the alloy used, the temperature window required for actuation may be suitable for in vivo applications. These applications are of great interest, given the demand for development of miniaturized medical instruments with increased functionality, e.g. for minimally invasive therapy. ${ }^{3}$ Such emerging applications of SMA actuation form the context of the present paper, which reports on research focused on the question how to most effectively employ SMAs in (micro-)actuated devices.

Many smart structures that utilize integrated SMA actuators make use of relatively simple actuator layouts, e.g. wires or helical springs. Clearly, for such layouts, understanding the behavior of a one-dimensional SMA wire or spring element is sufficient for effective design. However, further miniaturization of devices requires a higher degree of integration of functions, and consequently more complex SMA structures must be considered. Complex geometries, combined with the highly nonlinear SMA material behavior and the fact that multiple physical phenomena (electrical, thermal, mechanical) are involved, make the design of such structures a challenging task.

To assist designers of SMA structures, much effort has been devoted to computational modeling of SMA material behavior. However, next to computational modeling of the performance of given designs, systematic design optimization techniques can further facilitate the design process. ${ }^{4}$ Design optimization techniques have thus far not been explored to their full extent for SMA applications, despite the challenging design problems that exist. Although previous studies on optimization of SMA structures have reported groundbreaking advances, their scope has been rather limited as they relied on analytical models ${ }^{5}$ or simplifying assumptions. ${ }^{6}$ Therefore, we have studied more generic approaches suited for problems of realistic complexity. This paper reports the development of efficient topology and shape optimization techniques, specifically aimed at the design optimization of general SMA structures with complex geometries.

\footnotetext{
* Corresponding author. E-mail: M.Langelaar@tudelft.nl, Telephone +31 152786506
} Proc. of SPIE Vol. 6525, 65250W, (2007) · 0277-786X/07/\$18 · doi: 10.1117/12.715558 
This paper is organized as follows: firstly, in Sect. 2, the considered SMA material is introduced, and the way the constitutive model affects the complexity of the optimization process is discussed. Furthermore, an outline is given of the SMA constitutive model considered in this research. Subsequently, Sect. 3 considers topology optimization of SMA structures. In topology optimization, the layout of the design is not determined $a$ priori using e.g. a parametric geometrical model. ${ }^{7}$ Instead, the aim of the optimization procedure is to find the optimal distribution of SMA material in a given design domain. This makes topology optimization a powerful tool for the initial design stage, where it can help to find promising layouts and design concepts. The basic concepts of the applied topology optimization procedure are treated in Sect. 3, and several numerical examples are given. Subsequently, Sect. 4 presents the shape optimization of SMA structures operated by Joule heating. A gripper and an active catheter are considered as representative examples of design cases of realistic, complex SMA devices. Design sensitivity analysis and gradient-based optimization algorithms are employed to realize an efficient optimization procedure. Finally, concluding remarks are given in Sect. 5.

\section{CONSTITUTIVE MODELING}

\subsection{Material selection and modeling considerations}

Considering the intended applications of this research, i.e. actuated miniature medical instruments, the focus is on the shape memory effect due to the R-phase/austenite transformation in Ni-Ti. This transformation is characterized by a particularly small hysteresis and excellent cyclic stability, which makes it well suited for many actuation applications. Experimental data obtained by Tobushi et al. ${ }^{8}$ of the stress-strain-temperature behavior of a Ti- $55.3 \mathrm{wt} \% \mathrm{Ni}$ alloy exhibiting the R-phase transformation is shown in Fig. 1. It can be seen that the hysteresis between loading and unloading behavior is practically negligible in a certain temperature range, in this case between 328 and $343 \mathrm{~K}$. This range can be modified by adjusting the heat treatment procedure, ${ }^{9}$ therefore the use of this active material in in vivo biomedical applications is a possibility. In this study, the focus is on this temperature range with negligible hysteresis, and the validity of the material model discussed in this section is restricted to that temperature range.

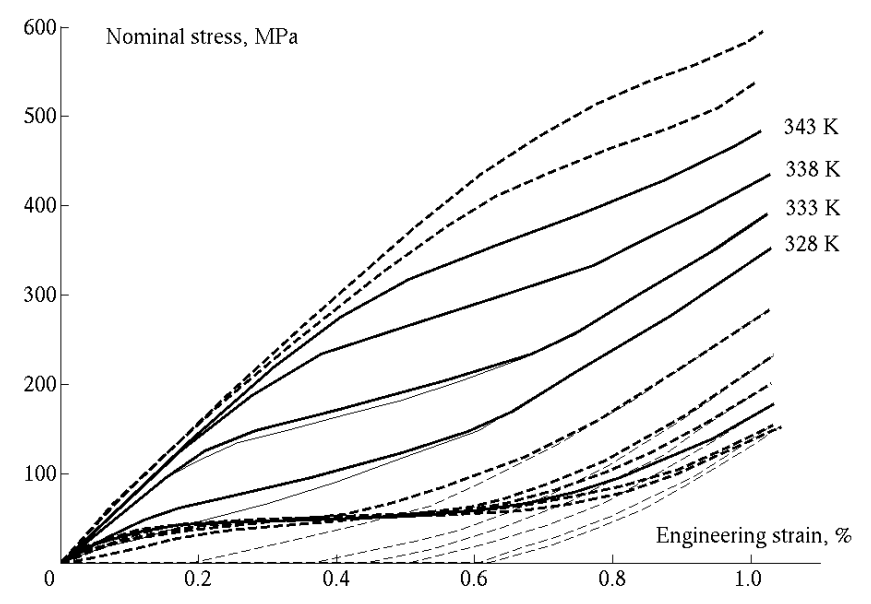

Figure 1. Stress-strain curves at various temperatures of a Ni-Ti alloy ${ }^{8}$. Thin lines are unloading curves.

In the formulation of a computational constitutive model, representing the experimentally observed material behavior illustrated in Fig. 1, it is important, in the context of this research, to not only consider the accuracy of the representation, but also to address the suitability of the model for subsequent design optimization. Design optimization generally is an iterative process that requires many evaluations of the analysis model. Thus, to make optimization approaches feasible in an engineering practice, it is important to consider the efficiency of the analysis model. In the present work, as a first simplification, the dynamic response of the actuator is neglected, and a quasistatic simulation is performed. This is sufficient in many applications where the response speed is not critical. 
A second aspect that is of great importance in design optimization is the complexity of the sensitivity analysis. Sensitivities, i.e. derivatives of responses with respect to design variables, allow the use of efficient gradient-based optimization algorithms. ${ }^{4}$ In history-dependent models, sensitivity analysis is much more involved than in the history-independent case. ${ }^{10}$ The internal variables used in most of the existing SMA models render those models history-dependent. In case of the material behavior considered in this research, the hysteresis that leads to a history-dependent response is particularly small. By neglecting the hysteresis of the R-phase transformation in the selected temperature range, it turns out to be possible to eliminate internal state variables, resulting in a history-independent model. In that case, sensitivity analysis can be carried out in a single step, after completing the analysis, without the need to account for every increment during the evolution of the model over time.

\subsection{SMA constitutive model}

A three-dimensional model has been developed to enable the analysis of general SMA structures for the material behavior described in Sect. 2.1. An outline of the modeling philosophy and the general structure of the model is given here. For further details on its formulation the reader is referred to a dedicated paper. ${ }^{11}$

The model is based on a generalization of a one-dimensional model of the experimental stress-strain-temperature curves shown in Fig. 1. Essentially, a piecewise linear stress-strain function is fitted to the experimental data, consisting of parts describing the austenite, transition and R-phase regions of the curve. In order to obtain a more accurate fit, selected coefficients of the piecewise linear segments are chosen to vary linearly with temperature. This results in a model with the following structure:

$$
\sigma_{x x}=\left\{\begin{array}{lll}
\varepsilon_{x x} \leq \varepsilon_{1}(T) & : \sigma_{0}=E_{A} \varepsilon_{x x} \\
\varepsilon_{1}(T)<\varepsilon_{x x} \leq \varepsilon_{2}(T) & : \quad \sigma_{1}=E_{T}(T)\left(\varepsilon_{x x}-\varepsilon_{1}(T)\right)+\sigma_{0}\left(\varepsilon_{1}(T)\right) \\
\varepsilon_{x x}>\varepsilon_{2}(T) & : \sigma_{2}=E_{R}\left(\varepsilon_{x x}-\varepsilon_{2}(T)\right)+\sigma_{1}\left(\varepsilon_{2}(T)\right)
\end{array}\right.
$$

Here $\sigma_{x x}$ and $\varepsilon_{x x}$ represent the one-dimensional stress and strain, respectively, $T$ denotes the temperature, $E_{A}$ and $E_{R}$ are the austenite and R-phase Young's moduli, $E_{T}$ is the apparent modulus during the phase transition, and $\varepsilon_{1}$ and $\varepsilon_{2}$ are strains that separate the distinct sections of the curve. Values for the coefficients used in this model are obtained through curve fitting. A detailed description of this model, together with a review of previously reported one-dimensional R-phase transformation models is given in an earlier paper. ${ }^{12}$

The generalization of this SMA model to a three dimensional setting is based on two additional experimental observations: the pressure-insensitivity of the the R-phase transformation and the isochoric nature of the transformation strain. These aspects are incorporated in the model through the use of a scalar strain measure $\varepsilon_{e}$ linked to the distortional strain energy, which affects a varying shear modulus $G$. The resulting stress-strain relation is consequently given by:

$$
\boldsymbol{\sigma}=\left(K \mathbf{K}+2 G\left(\varepsilon_{e}\right) \mathbf{G}\right) \varepsilon
$$

Here $K$ is the bulk modulus, $\boldsymbol{\sigma}$ and $\varepsilon$ are the second Piola-Kirchhoff stress and Green-Lagrange strain in vector notation, and matrices $\mathbf{G}$ and $\mathbf{K}$ in the preceding equation are given by:

$$
\mathbf{G}=\frac{1}{3}\left[\begin{array}{cccccc}
2 & -1 & -1 & 0 & 0 & 0 \\
-1 & 2 & -1 & 0 & 0 & 0 \\
-1 & -1 & 2 & 0 & 0 & 0 \\
0 & 0 & 0 & 3 & 0 & 0 \\
0 & 0 & 0 & 0 & 3 & 0 \\
0 & 0 & 0 & 0 & 0 & 3
\end{array}\right], \quad \mathbf{K}=\left[\begin{array}{llllll}
1 & 1 & 1 & 0 & 0 & 0 \\
1 & 1 & 1 & 0 & 0 & 0 \\
1 & 1 & 1 & 0 & 0 & 0 \\
0 & 0 & 0 & 0 & 0 & 0 \\
0 & 0 & 0 & 0 & 0 & 0 \\
0 & 0 & 0 & 0 & 0 & 0
\end{array}\right]
$$

Furthermore, the effective strain is defined as $\varepsilon_{e}^{2}=(2 / 3) \varepsilon^{T} \mathbf{G} \varepsilon$. This effective strain $\varepsilon_{e}$ is used to extend the one-dimensional model to the three-dimensional setting, in a way that is consistent with the experimentally observed R-phase transformation characteristics. For a constant shear modulus $G$, normal isotropic linear elastic behavior is retrieved. Further details on the formulation of this model and a description of the plane stress case can be found in a dedicated paper. ${ }^{11}$ Of importance in the present discussion is the fact that this model is history-independent, and therefore suited for efficient sensitivity analysis and design optimization. The model 
has been implemented in plate and shell elements, which are used in the optimization procedures discussed in Sections 3 and 4 .

The implementation has been verified using the available experimental data, as illustrated in Fig. 2. Because of the lack of firm experimental evidence regarding multi-dimensional, non-proportional loading it is presently not possible to perform further validation studies. However, the known main characteristics of the R-phase transformation are included, and therefore this model is presumed to capture the main effects of the constitutive behavior which are relevant for performing design optimization studies.

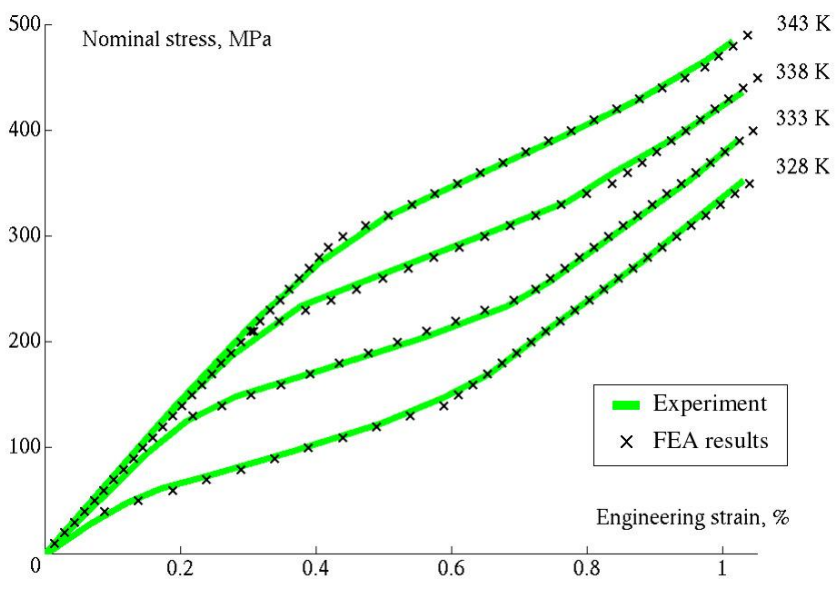

Figure 2. Experimental stress-strain curves at various temperatures ${ }^{8}$ together with finite element results obtained using the proposed SMA material model.

\section{TOPOLOGY OPTIMIZATION}

\subsection{Concept and methodology}

The aim of topology optimization is to find the optimal material distribution in a given domain to fulfill a certain objective. The technique, initially developed for the design of linear elastic structures, has been applied to a wide variety of engineering problems. ${ }^{7}$ The conventional density-based approach basically consists of controlling the material parameters of elements in a finite element model, e.g. the Young's moduli, using density design variables. $^{7}$ In this way, in e.g. structural problems, the presence of void regions in the structure can be approximated by elements with very low stiffness. In problems involving geometric nonlinearities, however, the low stiffness elements present in the density-based approach suffer from excessive distortion and cause numerical instabilities. Also the fact that a suitable relation needs to be defined between material parameters and density design variables makes it nontrivial to apply the density-based method to problems involving nonlinear materials, such as SMAs.

The Element Connectivity Parametrization (ECP) approach recently proposed by Yoon and Kim ${ }^{13}$ presents an alternative to the conventional density-based method, which avoids the mentioned disadvantages. In this ECP approach, not the material properties of elements, but the connectivity between the elements is controlled by design variables. This is implemented by introducing zero-length links between elements, as illustrated in Fig. 3. By controlling the link stiffnesses with design variables, different topologies can be realized. In order to prevent an increase in the number of system degrees of freedom, static condensation is applied to each element and its associated links. Due to this, in fact the links never need to be inserted in the mesh, but element stiffness matrices $\mathbf{K}_{e}$ are adapted using the following relation:

$$
\mathbf{K}_{e}^{C}=k_{L} \mathbf{I}-k_{L}^{2}\left[k_{L} \mathbf{I}+\mathbf{K}_{e}\right]^{-1}
$$


Here $\mathbf{K}_{e}^{C}$ represents the condensed stiffness matrix of the patch consisting of an element and its associated links with link stiffness $k_{L}$, as shown in Fig. 3, and $\mathbf{I}$ is the identity matrix. By replacing $\mathbf{K}_{e}$ by $\mathbf{K}_{e}^{C}$ in this way, the ECP approach can be conveniently implemented on element level.

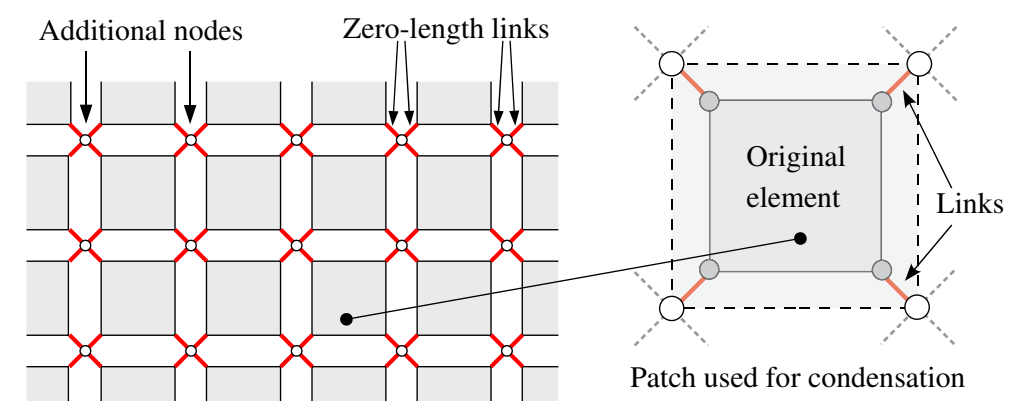

Figure 3. Layout of elements and zero-length links (shown here with finite length) used in the element connectivity parametrization approach.

In the outlined ECP approach, a key aspect is that elements maintain their nominal material properties, and only links are affected by design variables. As a result, problems with excessive distortion of weak elements no longer occur, and also the definition of a specific material interpolation for nonlinear material models is no longer necessary. This also makes that sensitivity analysis becomes straightforward, since only the link stiffnesses are affected by the design variables. Consequently, differentiation of material models is avoided. These advantages make the ECP method a very suitable approach for topology optimization of SMA structures. Its effectiveness is illustrated in the following section. A more technical discussion of the ECP method and its application to SMA topology optimization is beyond the scope of the present paper, and for this the interested reader is referred to dedicated articles. ${ }^{13-15}$

\subsection{Application to SMA actuator design}

Using the SMA material model presented in Sect. 2, and the ECP topology optimization approach outlined in Sect. 3.1, topology optimization of an SMA actuator has successfully been carried out. The problem considered is the design of a planar SMA structure that yields the largest in-plane displacement difference for a given constant load, when the temperature $T$ of the structure is varied homogeneously between 328 and $343 \mathrm{~K}$. Thus, the objective function $f$ for this optimization problem is defined as:

$$
f=\max \left|U_{T=328 \mathrm{~K}}-U_{T=343 \mathrm{~K}}\right| .
$$

The geometry of the design domain considered and the boundary conditions used are shown in Fig. 4(a). This problem only serves as an example to illustrate the possibilities of the described topology optimization procedure. It is straightforward to extend the technique to problems involving different geometries, loads and boundary conditions. In this case, symmetry of the design and its behavior is enforced, thus only half the domain was used in the optimization process. The output displacement $U$ considered in the present case is the vertical displacement at the point where the force $\mathrm{F}$ is applied.

A regular mesh consisting of 4000 standard four-node isoparametric quadrilateral plane stress finite elements was used. Design variables are associated to each node of the mesh, and the average value of these nodal design variables determines the link stiffness used in each patch. This nodal design variable approach leads to a continuous material distribution, and is adopted here to suppress numerical artifacts in the solution, such as checkerboard patterns and one-node hinges. ${ }^{7,16}$ In contrast to usual topology optimization formulations, no mass constraint is needed, as the SMA actuator topology optimization problem has the property to regulate the used amount of material by itself. 
The deformation of the SMA structure in the low and high temperature cases is evaluated using nonlinear finite element analysis. An adaptive incremental-iterative solution process is applied. In case the design changes are relatively small $(<2.5 \%)$, the solution process is started from the displacement field of the previous design, in order to reduce the overall computational cost. The optimizer used for the topology optimization is the Method of Moving Asymptotes (MMA), ${ }^{17}$ a gradient-based optimization algorithm. Gradients are computed using adjoint sensitivity analysis. ${ }^{13,15}$ Due to the history-independent nature of the used SMA material model (see Sect. 2) combined with the ECP approach, this only involves a relatively small computation after convergence of the analysis.

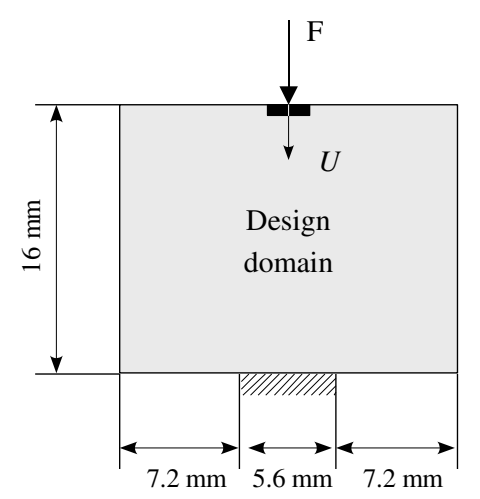

(a)

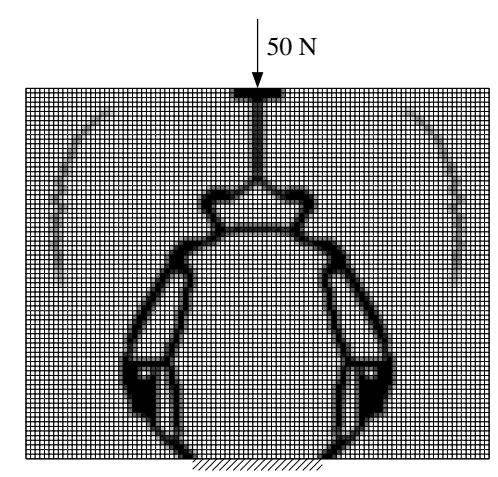

(b)

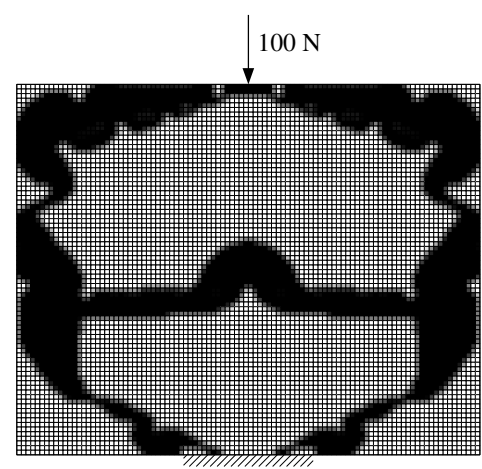

(c)

Figure 4. (a) Design domain and boundary conditions for the SMA topology optimization problem. (b,c) Topology optimization results of SMA thermal actuators for different load levels, obtained using the ECP topology optimization formulation. Solid and void regions are represented by black and white, respectively.

The optimization process is started from a uniform design configuration, where all design variables are set to an intermediate value representing $50 \%$ material. The optimization process continues to update the design variables until no further significant change in the objective function or the design is found. The resulting SMA actuator designs for two cases with different applied load levels are shown in Fig. 4(b) and (c). The obtained strokes are $0.12 \mathrm{~mm}$ for the $50 \mathrm{~N}$ case, and $0.55 \mathrm{~mm}$ for the $100 \mathrm{~N}$ case. Note that rather complex structures are generated, even for this apparently simple loadcase. The topologies obtained for the different load levels are very dissimilar, which reflects the nonlinearity of the underlying SMA material behavior. Larger loads typically result in structures with thicker members, which relates to the fact that the R-phase/austenite transformation is most effectively used in a particular strain range. Thicknesses are adapted in order to achieve similar strain levels in the material. In the designs generated in this way, the shape memory effect based on the R-phase/austenite transformation is converted most effectively to a large stroke of the actuator.

To conclude this section, two final remarks must be made about the accuracy of the analysis and the optimality of the obtained designs. Firstly, in the final designs shown in Fig. 4, some members are modeled by only a few elements in transverse direction. This may not be sufficiently accurate for a good prediction of strains and stresses in the material. This problem is often encountered in topology optimization, but it can easily be resolved by raising the number of elements per design patch or by using higher order elements. Finding the best compromise between cost and accuracy is up to the designer. Alternatively, the use of high-fidelity models can also be postponed to a second design phase, in which the result of the topology optimization process is used as a basis for a subsequent shape optimization.

Secondly, it should be noted that it is not possible to verify that the obtained designs are the absolute optimal structures for the given problem. The nonlinearity of the SMA material behavior and the complexity of the topology optimization process make it likely that the problem is nonconvex, and multiple local optima exist. In general, for such problems, no efficient methods exist that guarantee convergence to the global optimum. However, the proposed technique is shown to be able to automatically generate well-performing structures, that can serve as a basis for a more detailed design study, such as those presented in the next section. 


\section{SHAPE OPTIMIZATION}

\subsection{Shape optimization procedure}

Once a promising design concept has been established, for instance by topology optimization, its performance can be optimized by finding the best values for various shape parameters. In this section, two examples are discussed which illustrate the possibilities shape optimization offers for the design optimization of SMA structures of realistic complexity. The structures, a miniature gripper and an active catheter, both make use of Joule heating to locally increase the temperature of the SMA material. The resulting change in material properties interacts with internal prestress present in the structures, leading to deformations. In both cases, the objective of the optimization is to maximize the deformation in a functional mode, while restrictions are placed on the maximum temperatures and strains, i.e.:

$$
\begin{array}{ll}
\max & \left|U_{T=T_{\text {low }}}-U_{T=T_{\text {high }}}\right| \\
\text { subject to } & T \leq T_{\max } \\
& \varepsilon \leq \varepsilon_{\max }
\end{array}
$$

In order to evaluate the performance of the SMA structure, a sequentially coupled electrical, thermal and mechanical finite element analysis is performed. The mechanical analysis involves geometrical and physical nonlinearities, and makes use of the material model described in Sect. 2. The electrical power dissipated into the structure serves as a heat source for the heat transfer simulation, which results in a certain temperature distribution. The local temperature values are input for the SMA material model used in the mechanical analysis.

In order to reduce the computational cost of the procedure, and to thus make shape optimization feasible for SMA design problems of realistic complexity, it is attractive to make use of gradient-based optimization techniques. Gradient-based optimization algorithms generally require much fewer iterations to reach convergence, in comparison to algorithms that do not exploit gradient information. However, it is essential that design sensitivities can be computed at low computational cost, to achieve an overall efficient procedure. The historyindependent nature of the SMA material model described in Sect. 2 allows for efficient sensitivity analysis, and various options are formulated, discussed and compared in detail in recent studies. ${ }^{18,19}$ It turns out that the semi-analytical approach provides the most efficient way to compute sensitivities for the considered class of problems, but also the finite difference approach in combination with fast reanalysis is attractive. The latter requires only slightly more computational effort, but its implementation is far less involved. In the examples discussed here, the gripper optimization makes use of semi-analytical sensitivity analysis, and the finite difference approach is adopted in the active catheter optimization study.

\subsection{Miniature SMA gripper}

The design concept for the considered miniature SMA gripper is depicted in Fig. 5(a). The gripper consists of an identical top and bottom arm made of folded Ni-Ti plates, and is intended to make use of the shape memory behavior due to the R-phase/austenite transformation, which allows this gripper to operate in a relatively narrow temperature window. To make use of the shape memory effect, an initial deformation is applied to generate a prestress in the material. Starting from the undeformed configuration shown in Fig. 5(a), the ends of the outer plates are pinched toward the inner plates during assembly. In this situation, the equilibrium configuration of each arm can be changed by changing the temperature of either the inner or outer plates. Joule heating is used for this purpose, and to guide the electrical current through individual plates a slit is present along the length of each plate. Heating the inner plates will cause the tip ends to move apart, opening the gripper. Similarly, heating the outer plates has the opposite effect. In the closed configuration, clamping forces of $100 \mathrm{mN}$ are applied in $z$-direction at the tips of the gripper.

Because of symmetry, only a quarter of the gripper needs to be modeled: in this case half the top arm is used. This part together with the parametrization of the geometry is shown in Fig. 5(b). Seven design variables have been selected for this design problem: the six geometrical parameters indicated in Fig. 5(b), and the voltage applied to operate the gripper. Further geometrical details of the miniature gripper and the performed optimization study can be found in a dedicated paper. ${ }^{20}$ 


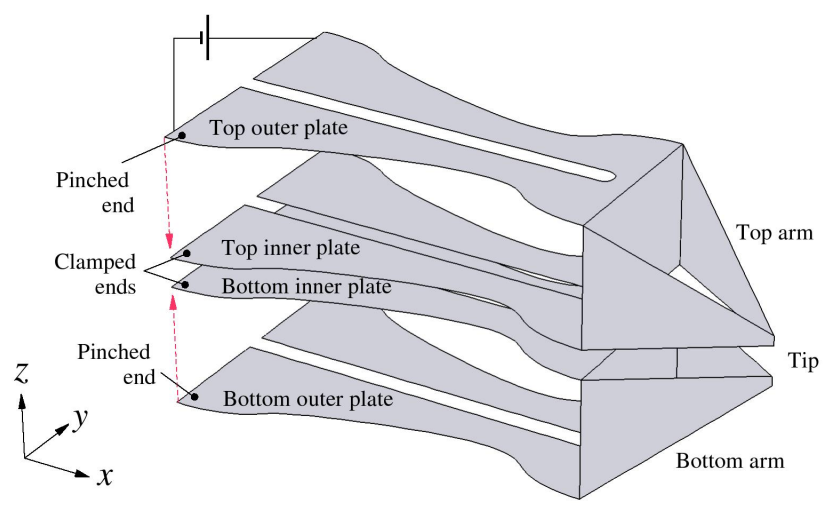

(a)

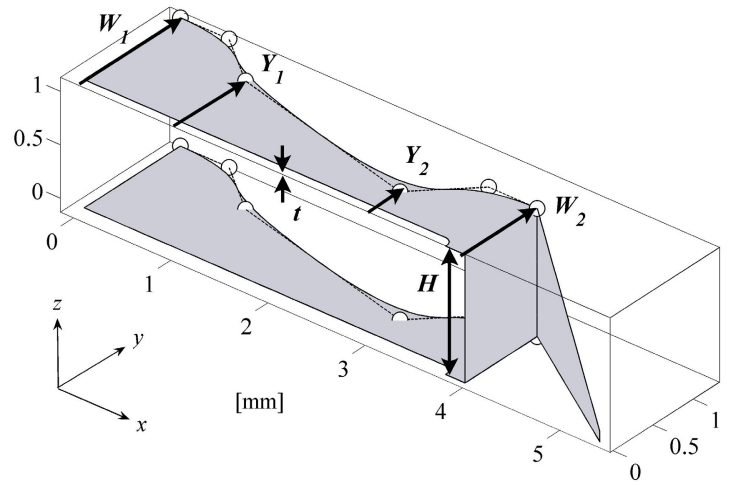

(b)

Figure 5. (a) Conceptual gripper geometry in the undeformed configuration. (b) Geometrical design variables used in the shape optimization procedure.

For the optimization, the gradient-based SQP optimization algorithm has been applied, using the implementation in the Matlab Optimization toolbox (Version 2.1). In order to enable grasping the widest possible range of objects, the objective of the optimization is to maximize the distance the gripper tips travel between the open and closed configuration. From three different starting points, the same optimum design was obtained in each case, using 17 design evaluations on average. In an earlier study, using an optimization algorithm that does not use gradient information, and only accounting for 5 design variables, hundreds of function evaluations were required. ${ }^{21}$ This illustrates the superior efficiency of the proposed approach. The temperature distribution on the obtained optimal design, in open and closed configurations, is depicted in Fig. 6. With operating temperatures restricted to the range $328-338 \mathrm{~K}$, the obtained maximum jaw opening was found to be $0.92 \mathrm{~mm}$.
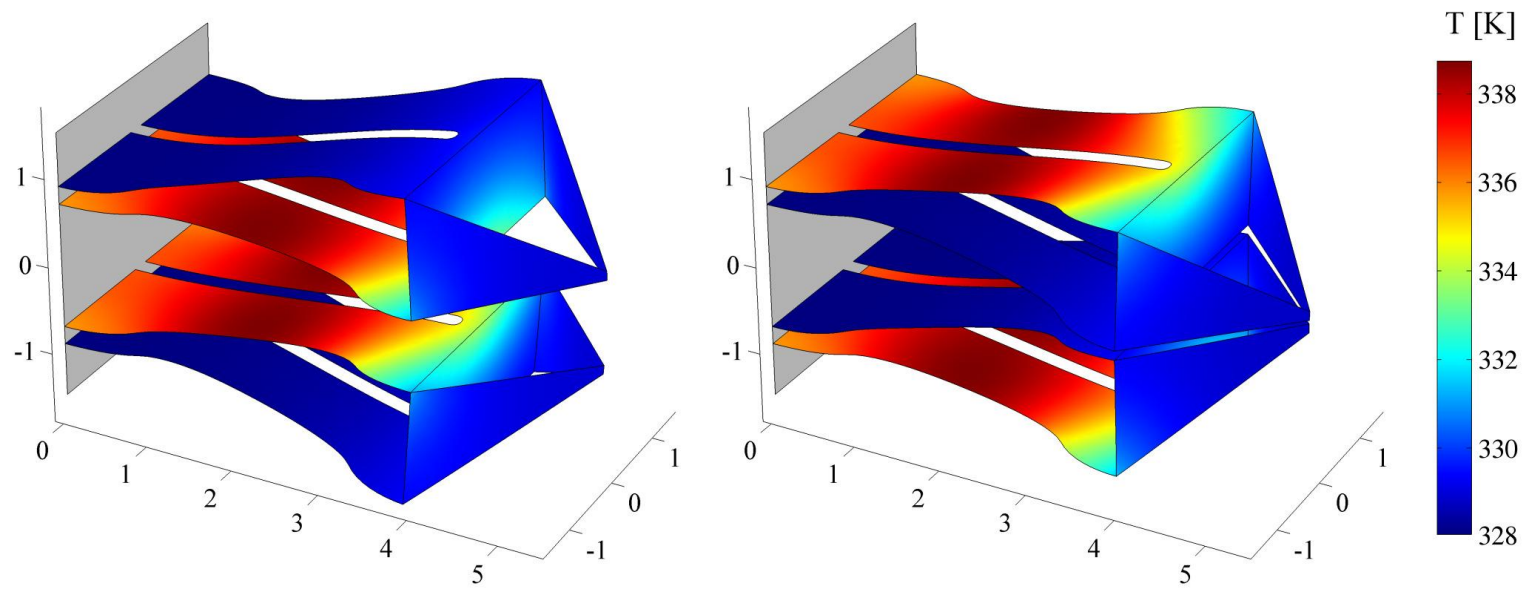

Figure 6. Temperature distribution $[\mathrm{K}]$ on the optimal miniature SMA gripper in the open and closed configuration.

\subsection{SMA active catheter}

The second shape optimization case discussed in this paper is the design of a steerable catheter, i.e. a catheter equipped with integrated SMA actuation sections that enable controlled bending in any direction. Catheterization involves insertion of a long, thin instrument in a branching network of bloodvessels (Fig. 7(a)). Proper positioning of the instrument is far from trivial. Therefore, improvement of the steerability and controllability 
of catheters by integrating miniaturized distributed actuators (cf. Fig. 7(b)) has attracted considerable interest. $^{22-24}$ For reasons discussed in Sect. 2, SMA actuation based on the R-phase/austenite transformation is well suited for this application. However, the strains that can be obtained by this transformation are limited, and therefore the shape memory effect must be exploited to its full extent in order to create functional devices. Gradient-based shape optimization is employed here to efficiently and systematically maximize the bending performance of several SMA active catheter design concepts.

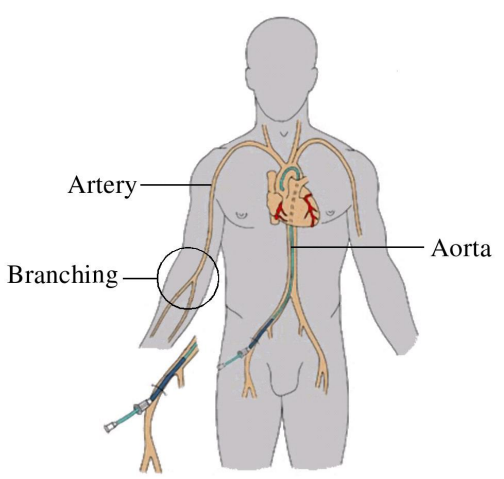

(a)

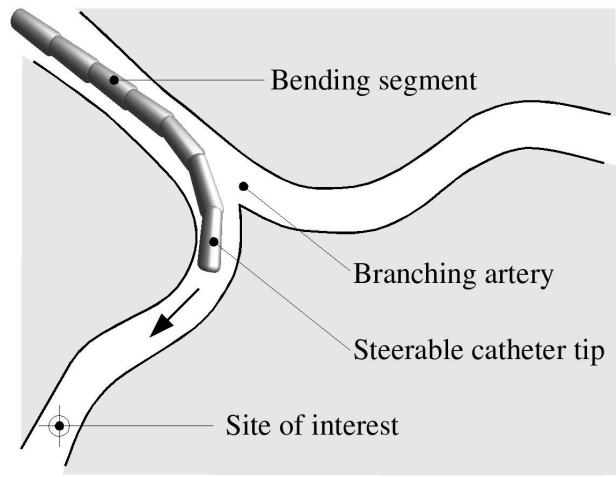

(b)

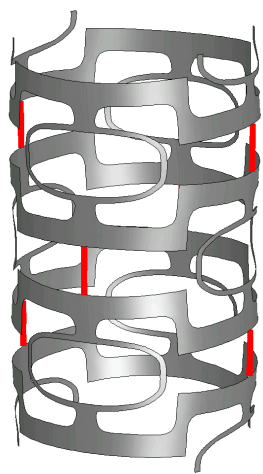

(c)

Figure 7. (a) Cardiac catheterization procedure. (b) Schematic illustration of an active catheter in use. (c) Considered SMA active catheter concept.

A segment of the active catheter concept considered in this section is depicted in Fig. 7(c). It consists of a structure laser-cut out of a Ni-Ti tube, in which after axial stretching spacers have been inserted to preload the structure. By locally heating sections of this structure, through application of different voltages to strategically placed distributed electrodes, the structure can be made to bend in a desired direction. The layout of the SMA structure is such, that bending in two orthogonal planes is possible, and by combining these the device can bend in any transverse direction.

A key consideration for in vivo use of this device is the maximum operating temperature. Based on maximum allowable temperatures for blood vessel wall tissues (squamous epithelial cells) and blood itself, ${ }^{25,26} 49^{\circ} \mathrm{C}(322$ $\mathrm{K}$ ) is used as a safe upper limit in this study. This would rule out the use of the material presented in Sect. 2, however, since its transformation temperatures can be modified by heat treatment, ${ }^{9}$ it is assumed that the material behavior observed in the range $328-340 \mathrm{~K}$ can also be achieved in a range of $310-322 \mathrm{~K}\left(37-49^{\circ} \mathrm{C}\right)$. Similar to the miniature gripper discussed in Sect. 4.2, the performance of the catheter is evaluated by means of a sequentially coupled electrical, thermal and mechanical finite element analysis, using again the material model outlined in Sect. 2. The periodicity and symmetry of the design concept allows consideration of only a small segment of the entire structure. A detailed discussion of the modeling of the catheter can be found in a previous paper. ${ }^{27}$

To evaluate the ultimate performance limits of various concepts, shape optimization of three active catheter design concepts has been performed, using the approach discussed in Sect. 4.1. Finite difference sensitivity analysis was employed, in combination with fast reanalysis. Perturbed designs are evaluated by starting the nonlinear mechanical analysis from the final nominal configuration, thus achieving significant savings in computational effort. Five design variables were considered in this exploratory study: the tube wall thickness, the applied voltage, the size of the spacers, and two variables controlling the shape of the used structural pattern. The maximum operating temperature was constrained to $322 \mathrm{~K}$ in an averaged sense, using Kreisselmeier-Steinhauser constraint aggregation. ${ }^{28}$ Furthermore, in order to remain within the range of the modeled material behavior, the maximum material strain was limited to $1 \%$.

Results of the optimization, performed again using the SQP optimization algorithm, are shown in Fig. 8. The diameter of the catheter has been kept constant at $1 \mathrm{~mm}$, and three concepts with different active segment layouts were optimized independently. In this case, on average 21 evaluations were required to converge to the 
optimum designs. Note that maximum temperatures locally exceed the $322 \mathrm{~K}$ temperature limit, but this is tolerable as it only occurs in a very small fraction of the structure. An earlier design, obtained by a trail and error approach, ${ }^{27}$ reached a bending radius of $35.2 \mathrm{~mm}$. The achieved minimum bending radii for the designs shown in Fig. 8 were 20.0, 14.1 and $14.2 \mathrm{~mm}$, respectively, which are considerable improvements. This degree of bending capability could certainly be useful for practical applications. Still, based on this success, further studies with a larger set of geometrical parameters are planned to determine the ultimate limits.
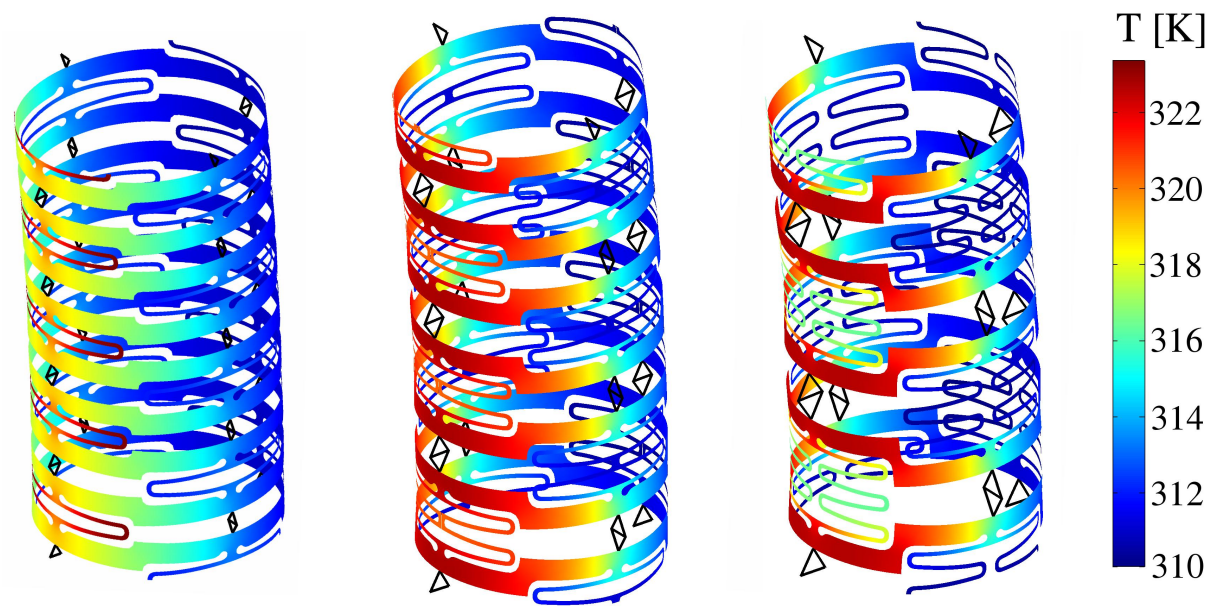

Figure 8. Temperature distributions at maximum bending in parts of the optimized deformed catheter structure with $1 \mathrm{~mm}$ tube diameter, for the three considered design concepts.

\section{CONCLUDING REMARKS}

This paper has presented several examples of the successful application of versatile design optimization techniques to the design of SMA active structures of realistic complexity, with a focus on the shape memory behavior due to the R-phase/austenite transformation in Ni-Ti. Topology optimization was shown to be able to generate well-performing designs without the need to predefine a certain geometry. This technique could be applied to generate promising initial design layouts, or to improve existing designs. The use of the element connectivity parametrization approach avoided problems with excessive element distortion and proved easy to combine with nonlinear material models.

Furthermore, for detailed optimization of designs using high-fidelity finite element modeling, an efficient gradient-based shape optimization approach was presented. By making use of computationally inexpensive sensitivity analysis, enabled by considering suitability for optimization at the formulation of the SMA material model, the number of costly finite element simulations required for optimization was kept to a minimum. Both for a miniature gripper and an active catheter, well-performing designs were obtained in a systematic way, with modest computational effort. The presented optimization approaches are applicable to a wide variety of SMA devices, and have the potential to be a valuable addition to the set of computational techniques available to the designers of SMA smart structures.

\section{ACKNOWLEDGMENTS}

The Dutch MicroNed programme is acknowledged for financially supporting this research. Furthermore, the authors thank Prof. Krister Svanberg for providing his implementation of his optimization algorithm "Method of Moving Asymptotes". The work on topology optimization of SMA structures was carried out at the Multiscale Design Center of Seoul National University, Seoul, Korea, in close collaboration with Prof. Y.Y. Kim and Dr. G.H. Yoon. Their support is also gratefully acknowledged. 


\section{REFERENCES}

1. K. Otsuka and C. Wayman, eds., Shape Memory Materials, Cambridge University Press, 1998.

2. T. Duerig, K. Melton, D. Stöckel, and C. Wayman, eds., Engineering Aspects of Shape Memory Alloys, Butterworth-Heinemann, London, 1990.

3. F. Tendick, S. Sastry, R. Fearing, and M. Cohn, "Applications of micromechatronics in minimally invasive surgery," IEEE-ASME Transactions on Mechatronics 3, pp. 34-42, March 1998.

4. R. Haftka and Z. Gürdal, Elements of structural optimization, Kluwer, Dordrecht, 1992.

5. T. Lu, J. Hutchinson, and A. Evans, "Optimal design of a flexural actuator," Journal of the Mechanics and Physics of Solids 49, pp. 2071-2093, September 2001.

6. M. Kohl, K. Skrobanek, and S. Miyazaki, "Development of stress-optimized shape memory microvalves," Sensors and Actuators, A: Physical 72, pp. 243-250, February 1999.

7. M. Bendsoe and O. Sigmund, Topology Optimization - Theory, Methods and Applications, Springer-Verlag, Berlin, 2003.

8. H. Tobushi, K. Tanaka, K. Kimura, T. Hori, and T. Sawada, "Stress-strain-temperature relationship associated with the R-phase transformation in Ti-Ni shape memory alloy," JSME International Journal Series I - Solid Mechanics Strength of Materials 35, pp. 278-284, July 1992.

9. T. Sawada, H. Tobushi, K. Kimura, T. Hattori, K. Tanaka, and P. Lin, "Stress-strain-temperature relationship associated with the R-phase transformation in TiNi shape memory alloy (influence of shape memory processing temperature)," JSME International Journal Series A: Mechanics and Material Engineering 36(1), pp. 395-401, 1993.

10. M. Kleiber, H. Antúnez, T. Hien, and P. Kowalczyk, Parameter Sensitivity in Nonlinear Mechanics: Theory and Finite Element Computations, John Wiley \& Sons Ltd., 1997.

11. M. Langelaar and F. van Keulen, "Modeling of shape memory alloy shells for design optimization.." Accepted for publication in Computers and Structures, 2007.

12. M. Langelaar and F. van Keulen, "A simple R-phase transformation model for engineering purposes," $M a$ terials Science and Engineering: A - Structural Materials: Properties, Microstructure and Processing 378, pp. 507-512, July 2004.

13. G. Yoon and Y. Kim, "Element connectivity parameterization for topology optimization of geometrically nonlinear structures," International Journal of Solids and Structures 42(7), pp. 1983-2009, 2005.

14. G. Yoon, Y. Kim, M. Langelaar, and F. van Keulen, "Theoretical aspects of the internal element connectivity parameterization approach for topology optimization." Submitted to Int. J. Num. Meth. Eng., 2006.

15. M.Langelaar, G. Yoon, Y. Kim, and F. van Keulen, "Topology optimization of shape memory alloy actuators using element connectivity parameterization," in 6th World Congress on Structural and Multidisciplinary Optimization, Rio de Janeiro, Brazil, May 30 - June 3, 2005.

16. K. Matsui and K. Terada, "Continuous approximation of material distribution for topology optimization," International Journal for Numerical Methods in Engineering 59, pp. 1925-1944, April 2004.

17. K. Svanberg, "MMA - method of moving asymptotes - a new method for structural optimization," International Journal for Numerical Methods in Engineering 24, pp. 359-373, 1987.

18. M. Langelaar and F. van Keulen, "Sensitivity analysis of shape memory alloy shells," in Third European Conference on Computational Mechanics, Lisbon, Portugal, 5-8 June, 2006.

19. M. Langelaar and F. van Keulen, "Sensitivity analysis of shape memory alloy shells.." Accepted for publication in Computers and Structures, 2007.

20. M. Langelaar and F. van Keulen, "Sensitivity analysis and optimization of a shape memory alloy gripper," in Proceedings of the Eighth International Conference on Computational Structures Technology, B. H. V. Topping, G. Montero, and R. Montenegro, eds., Civil-Comp Press, (Stirlingshire, United Kingdom), 2006. paper 202.

21. M. Langelaar and F. van Keulen, "Design optimization of shape memory alloy structures," in 10th AIAA/ISSMO Multidisciplinary Analysis and Optimization Conference, Albany, NY, 2004. AIAA paper 2004-4414. 
22. S. Kaneko, S. Aramaki, K. Arai, Y. Takahashi, H. Adachi, and K. Yanagisawa, "Multi-freedom tube type manipulator with SMA plate," Journal of Intelligent Material Systems and Structures 7(3), pp. 331-335, 1996.

23. K. Park and M. Esashi, "A multilink active catheter with polyimide-based integrated CMOS interface circuits," Journal of Microelectromechanical Systems 8, pp. 349-357, December 1999.

24. T. Mineta, T. Mitsui, Y. Watanabe, S. Kobayashi, Y. Haga, and M. Esashi, "Batch fabricated flat meandering shape memory alloy actuator for active catheter," Sensors and Actuators A: Physical 88, pp. 112-120, February 2001.

25. E. Ng and L. Chua, "Prediction of skin burn injury. part 2: Parametric and sensitivity analysis," Proceedings of the Institution of Mechanical Engineers Part H - Journal of Engineering in Medicine 216(3), pp. 171-183, 2002.

26. D. Herron, R. Grabowy, R. Connolly, and S. Schwaitzberg, "The limits of bloodwarming: Maximally heating blood with an inline microwave bloodwarmer," Journal of Trauma - Injury, Infection and Critical Care 43(2), pp. 219-228, 1997.

27. M. Langelaar and F. van Keulen, "Modeling of a shape memory alloy active catheter," in 12th AIAA/ASME/AHS Adaptive Structures Conference, Palm Springs, CA, 2004. AIAA paper 2004-1653.

28. G. Kreisselmeier and R. Steinhauser, "Application of vector performance optimization to a robust control loop design for a fighter aircraft," International Journal of Control 37, pp. 251-284, 1983. 